\title{
Goodpasture's syndrome: treatment with plasmapheresis, immunosuppression, and anticoagulation
}

\author{
M LEVIN, S P A RIGDEN, J R PINCOTT, C M LOCKWOOD, T M BARRATT, AND \\ M J DILLON \\ Renal Unit and Department of Histopathology, The Hospital for Sick Children, Great Ormond Street, \\ London, and The Renal Unit, Hammersmith Hospital, Du Cane Road, London
}

SUMMARY We report 3 children with Goodpasture's syndrome. In 2 children the diagnosis was delayed, treatment began late, and they did not recover renal function. In the third child early diagnosis and intensive treatment with plasmapheresis, immunosuppression, and anticoagulation resulted in an initial return of renal function after a prolonged period of anuria. In this child, however, although there was no evidence of disease activity, further deterioration of renal function subsequently occurred.

Goodpasture's syndrome, a term describing the association of glomerulonephritis and lung haemorrhage $^{1}$ is an uncommon but frequently fatal illness. Although the term originally referred to this clinical association without any implications as to aetiology or pathogenesis, elucidation of the pathological processes $^{2-4}$ has allowed it to be defined more accurately by immunological ${ }^{45}$ and pathological features. ${ }^{6} 7$ The disorder is associated with antibodies to glomerular basement membrane ( $\alpha$-GBM) that cross react with alveolar basement membrane. ${ }^{478}$ The pathogenic role of these antibodies has been established by transfer experiments ${ }^{59}$ and they may be identified in the circulation by radioimmunoassay and by indirect immunofluorescence. ${ }^{9-11}$ The presence in the circulation of $\alpha-G B M$ together with the characteristic linear deposition of IgG along the glomerular basement membrane are the immunopathological hallmarks of the condition. ${ }^{5}$ These features enable auto-antibody mediated Goodpasture's syndrome to be distinguished as a specific condition from other pathological processes with both renal and pulmonary manifestations such as Wegener's granulomatosis, polyarteritis, and acute post-streptococcal glomerulonephritis with pulmonary oedema. Although simultaneous occurrence of renal and pulmonary manifestations were the diagnostic features of the syndrome as originally described, $\alpha$-GBM disease may occur with isolated renal or pulmonary involvement, and with both systems affected at different stages of the illness. ${ }^{512}$ It has been suggested that the term Goodpasture's syndrome should be replaced by the pathogenetically descriptive and broader definition, 'anti-basement membrane antibody induced glomerulonephritis and pulmonary haemorrhage'. We have, however, with others, ${ }^{13} 14$ retained the term Goodpasture's syndrome for reasons of brevity and current familiarity.

Goodpasture's syndrome (or anti GBM glomerulonephritis and pneumonitis) usually affects adults. Most series have shown a predominance of young men and we are not aware of any reported cases in children other than those alluded to by Anand ${ }^{15}$ and Siegler. ${ }^{16}$

Although the cause of the $\alpha$-GBM antibody production is unknown, exposure to hydrocarbon fumes and viral illnesses have been implicated as precipitating events. ${ }^{1718} \mathrm{~A}$ genetic predisposition has been established by the finding of a strong association of the human leucocyte antigen DRw2 with the disease, ${ }^{19}$ as well as by its occurrence in identical twins. ${ }^{20}$

The renal component is frequently characterised by a severe proliferative glomerulonephritis with extensive crescent formation. Although there is considerable variability in the rate of progression of the renal disease and some cases with focal glomerular involvement show only very slow deterioration, patients with an appreciable proportion of crescents have a uniformly bad prognosis. Most large series have found that between $44 \%$ and $55 \%$ of patients have died, with a large percentage of the survivors requiring long term dialysis and renal transplantation. ${ }^{513}$ 
After the recognition of the pathogenetic role of the $\alpha$-GBM antibody attempts were made to treat the disease with plasmapheresis coupled with immunosuppression, to remove physically the $\alpha-G B M$, and to prevent or diminish their production. Several published reports have shown that a considerable improvement in mortality may be achieved with control of lung haemorrhage, reduction in antibody level, and improvement in renal function; and plasmapheresis has become standard treatment for the disorder in adults. Recovery of renal function in patients with immunologically proved Goodpasture's syndrome is, however, extremely rare once total anuria has occurred.21-23

We report our experience with 3 children with Goodpasture's syndrome. The diagnosis in each case was confirmed by renal biopsy examination and the finding of circulating $\alpha$-GBM. In the first 2 patients delay in diagnosis and in initiating treatment, together with technical difficulties in treatment, resulted in failure to halt the progress of the disease, and permanent loss of renal function ensued. In the third patient early diagnosis and treatment with intensive plasmapheresis, immunosuppression, and anticoagulation resulted in an initial return of renal function after a prolonged period of anuria.

\section{Case reports}

Case 1. A previously well 4 year old girl was admitted to her local hospital in November 1977 with a 4 month history of loin pain, pallor, anorexia, and lethargy. There was no history of preceding infection, hydrocarbon exposure, or a family history of renal disease. She was anaemic and mildly uraemic with microscopic haematuria and proteinuria. Renal function deteriorated progressively over the next few weeks and 4 weeks later she was transferred to the Renal Unit at the Hospital for Sick Children.

On admission she was pale and normotensive, but had no other abnormal respiratory or other signs. Investigation results showed the following: haemoglobin concentration $7.5 \mathrm{~g} / \mathrm{dl}$; platelets $160 \times 10^{9} / 1$ $\left(160000 / \mathrm{mm}^{3}\right)$; white cell count $12.3 \times 10^{9} / 1$ $\left(12.3 \times 10^{3} / \mathrm{mm}^{3}\right.$ ) (neutrophils $80 \%$, lymphocytes $15 \%$, monocytes $2 \%$, eosinophils $2 \%$ ); fibrin degradation products and fibrinogen normal; erythrocyte sedimentation rate $70 \mathrm{~mm} / \mathrm{h}$; plasma creatinine $466 \mu \mathrm{mol} / 1(5.27 \mathrm{mg} / 100 \mathrm{ml})$; urea $23 \mathrm{mmol} / \mathrm{l}$ $(138.5 \mathrm{mg} / 100 \mathrm{ml})$; albumin $29 \mathrm{~g} / \mathrm{l}$; electrolytes normal; plasma $\mathrm{C} 3$ and $\mathrm{C} 4$ normal; antinuclear factors and anti-deoxyriboneucleic acid antibodies not detected; antistreptolysin $O$ titre $150 \mathrm{U} / \mathrm{ml}$; Coombs's test negative; no cold agglutinins or immune complexes detected; chest radiograph normal; urine showed microscopic haematuria, granular casts, and heavy proteinuria. A renal biopsy specimen showed an end stage glomerulonephritis with evidence of previous crescent formation in all glomeruli, and extensive tubular atrophy and interstitial fibrosis. Immunofluorescence showed diffuse linear deposition of IgG and smaller amounts of C3 in the capillary loops, but staining for IgA, IgM, CIq, and fibrin was negative.

A diagnosis of Goodpasture's syndrome was made on the basis of the typical linear deposition of IgG on immunofluorescence. This was confirmed by detection of high titres of circulating $\alpha$-GBM ( $>50 \%$; normal range $>12 \%$ binding of reference positive serum) using a sensitive solid phase radioimmunoassay. ${ }^{24}$ She was transferred to Hammersmith Hospital for plasmapheresis 2 weeks later. There were considerable difficulties in establishing adequate vascular access for plasmapheresis, initially via an arteriovenous shunt and subsequently through a femoral vein line. Attempts at plasmapheresis were abandoned after 2 exchanges of $500 \mathrm{ml}$. Treatment with prednisolone, azathioprine, and cyclophosphamide produced no improvement, and she died 6 weeks later.

Case 2. A previously well 10 year old girl develope haematuria and vomiting 1 day after the onset of sore throat and fever for which ampicillin had beex. prescribed. There was no other drug or hydrocarbo of exposure. She had 1 sister with acute lymphatic leukaemia and another with epilepsy, but no family history of renal disease. Urine output decreased over the next 2 days and she was admitted to hospital where she was found to be uraemic and oliguric. Renal function deteriorated over the next 3 days and she was transferred to the Renal Unit at the Hospital for Sick Children 6 days after the onset of symptoms.

On admission she was pale and normotensive, but there were no other abnormal physical signs. Results of investigations were as follows: haemoglobin 11 $\mathrm{g} / \mathrm{dl}$; white cell count $16.9 \times 10^{9} / 1\left(16.9 \times 10^{3} / \mathrm{mm}^{3}\right)$ (79\% neutrophils, $17 \%$ lymphocytes and $3 \%$ monocytes); coagulation studies normal; plasma 을 creatinine $400 \mu \mathrm{mol} / 1(4.5 \mathrm{mg} / 100 \mathrm{ml})$; urea $27 \stackrel{7}{7}$ $\mathrm{mmol} / 1(163 \mathrm{mg} / 100 \mathrm{ml})$; electrolytes normal. Her 을 urine contained numerous white cells, red cells, N granular casts, and there was heavy proteinuria. Her chest radiograph was normal. Plasma C3 was $\tilde{}$ low on 2 occasions $(40 \%$ and $17 \%$ of standard) $\underset{\omega}{N}$ but $\mathrm{C} 4$ and total haemolytic complement were 응 normal: antinuclear factor, anti-deoxyribonucleic 0 acid antibodies, and immune complexes were not $\stackrel{\mathbb{D}}{\overparen{D}}$ detected. Throat swab, blood cultures, and viral $\stackrel{\infty}{\rightarrow}$ serology and cultures were all negative, and her antistreptolysin $\mathrm{O}$ titre was not raised. 
The low $\mathrm{C} 3$ and preceding throat infection suggested a diagnosis of acute post-streptococcal glomerulonephritis and an initial period of fluid restriction and observation was undertaken. Renal function progressively deteriorated, however, and renal biopsy examination was performed 4 days after admission. After the biopsy examination she became increasingly uraemic and peritoneal dialysis began the following day.

The renal biopsy histology (Fig. 1) showed an endocapillary and an extracapillary proliferative glomerulonephritis with florid epithelial crescents in $80 \%$ of the glomeruli. There was insufficient material for immunofluorescence. Treatment for her crescentic nephritis began with prednisolone $40 \mathrm{mg} / \mathrm{m}^{2}$ per day, azothioprine $2 \mathrm{mg} / \mathrm{kg}$ per day, dipyridamole 10 $\mathrm{mg} / \mathrm{kg}$ per day, and heparin $100 \mathrm{U} / \mathrm{kg} 8$ hourly.

Since the cause of the crescentic nephritis was still unclear, blood was sent to the Hammersmith Hospital for $\alpha$-GBM titre and the result, received within 24 hours, showed an extremely high titre ( $>100 \%$ binding) establishing the diagnosis of Goodpasture's syndrome. Plasmapheresis began without delay (after 8 days of peritoneal dialysis) and was performed daily for the next 4 days, exchanging 1.2-2.0 litres of plasma on each occasion. Three days later the $\alpha$-GBM titre had fallen to one quarter of the original value but she developed peritonitis and abdominal wall cellulitis around the site of the peritoneal dialysis catheter, which had been resited because of drainage problems.

In view of the rapidly spreading infection further immunosuppression and plasmapheresis was felt to be hazardous, and she was transferred for haemodialysis. No further attempts were made to remove or suppress $\alpha$-GBM formation with plasmapheresis or immunosuppression and she has rernained in renal failure on long term haemodialysis.

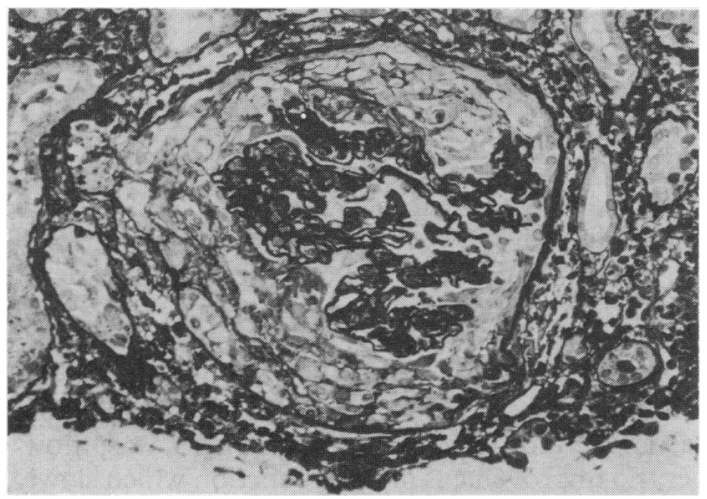

Fig. 1 Renal biopsy, case 2 ( $\times 450$-methenamine silver). The epithelial crescent surrounds the glomerular tuft.
Case 3. A previously well 7 year old girl developed diarrhoea and vomiting. There was no history of exposure to hydrocarbons or a preceding infective episode. She had suffered from episodes of mild wheezing and dyspnoea on exercise during the preceding year.

Diarrhoea stopped after 24 hours but she continued to vomit intermittently. Over the course of the next week she became increasingly lethargic, anorexic, and pale and she passed urine less frequently. Nine days after the onset of symptoms she was admitted to hospital. She was found to be anaemic, uraemic, and anuric and was therefore transferred to the Renal Unit at the Hospital for Sick Children on the following day.

On admission she was pale and drowsy and her blood pressure was $110 / 70 \mathrm{mmHg}$. She had a moist cough but no abnormal signs in the lungs or cardiovascular system and there were no other important findings. Initial investigation results were as follows: haemoglobin $7.4 \mathrm{~g} / \mathrm{dl}$, white cell count $16.6 \times 10^{9} / 1$ $\left(16.6 \times 10^{3} / \mathrm{mm}^{3}\right)(84 \%$ neutrophils, $11 \%$ lymphocytes and $3 \%$ monocytes); erythrocyte sedimentation rate $20 \mathrm{~mm} / \mathrm{h}$, platelets $500 \times 10^{9} / 1\left(500000 / \mathrm{mm}^{3}\right)$; coagulation studies, serum fibrin degradation products, and plasma fibrinogen normal; plasma creatinine $1300 \mu \mathrm{mol} / 1(14.7 \mathrm{mg} / 100 \mathrm{ml})$; urea 61 $\mathrm{mmol} / \mathrm{l}(367 \mathrm{mg} / 100 \mathrm{ml})$; electrolytes normal; albumin $26 \mathrm{~g} / 1$; plasma C3 normal; antinuclear factor, anti-deoxyribonucleic acid antibodies, and immune complexes not detected; antistreptolysin $\mathrm{O}$ titre 100 units $/ \mathrm{ml}$; viral serology negative. Her chest radiograph was normal initially but a repeat 3 days later showed a haze over the right lung with peribronchial interstitial shadowing. No urine was obtainable for examination.

Peritoneal dialysis began soon after admission. Because of a close clinical resemblance to our previous patient, blood was sent on the night of admission to the Hammersmith Hospital for estimation of $\alpha$-GBM titre. Twenty four hours later the result was received showing an extremely high titre of $\alpha$-GBM ( $>45 \%$ binding), and plasmapheresis, immunosuppression, and anticoagulation were begun immediately - without delaying treatment to perform a renal biopsy for diagnostic confirmation.

Plasmapheresis was performed using the Hemonetic cell separator. The procedure was accomplished via a percutaneous central line for the first 10 days, and thereafter through peripheral veins. She was started on treatment with prednisolone 60 $\mathrm{mg} / \mathrm{m}^{2}$, cyclophosphamide $3 \mathrm{mg} / \mathrm{kg}$ per day, and heparin $100 \mathrm{U} / \mathrm{kg} 8$ hourly. Her progress is illustrated in Fig. 2.

Plasmapheresis was undertaken daily for 10 days, 


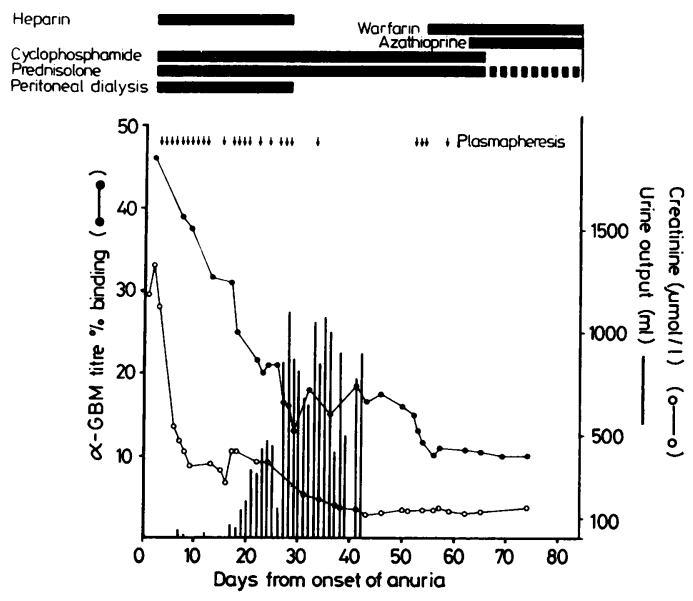

Fig. 2 Progress of patient in case 3 in relation to treatment.

Normal range for $\alpha$-GBM titre $<12 \%$ binding of reference positive serum. Urine output not measured after day 42.

exchanging 1.3-1.6 1 plasma (about twice the patient's plasma volume) on each occasion. The procedure was performed over 4-6 hours, and was well tolerated by the patient.

After the first 10 days of treatment, she remained anuric and the $\alpha$-GBM antibody titre on day 10 was still notably raised, although lower than pretreatment value. A more intensive effort was therefore made to remove the antibody. The volume of plasma exchanged on each occasion was increased to between 2.8 and 3.8 litres $(\approx 4$ times the patient's total plasma volume) and a further 11 exchanges were performed, taking between 6-8 hours on each occasion.

On day 16 of treatment, after the first 3 exchanges of 3.81 , the $\alpha$-GBM titre fell to within normal values and a small quantity of urine was passed. Thereafter there was a progressive increase in urine output and the antibody remained low. Dialysis was discontinued on day 24 of treatment and plasmapheresis stopped after 21 exchanges. A renal biopsy examination performed on day 27 of treatment showed crescentic nephritis affecting all glomeruli with the characteristic linear deposition of IgG on immunofluorescence, as shown in Fig. 3.

After the end of plasmapheresis renal function stabilised with plasma creatinine value of $100-200$ $\mu \mathrm{mol} / 1(1 \cdot 1-2 \cdot 2 \mathrm{mg} / 100 \mathrm{ml})$. Heparinization was scopped before renal biopsy examination, and after recovery from this warfarin was introduced for long term anticoagulation. $\alpha-G B M$ antibody remained low after stopping plasmapheresis until day 48 when it again became notably raised. This rise in

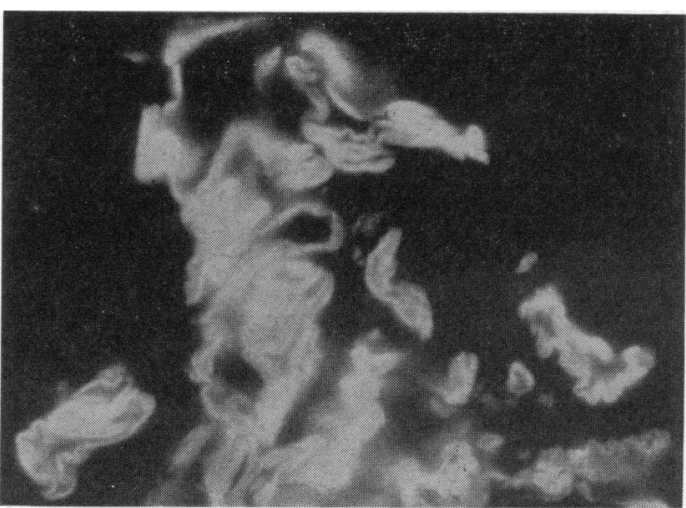

Fig. 3 Renal biopsy, case $3(\times 720)$. Immunofluorescence shows a diffuse linear deposition of IgG in glomerular capillary loops.

antibody titre was associated with an increase in the plasma creatinine concentration. Three more plasma exchanges were performed resulting in undetectable values of $\alpha-G B M$ in the plasma and a return of plasma creatinine to previous concentrations.

Cyclophosphamide was continued for 8 weeks and was then withdrawn and replaced by azathio prine, $2 \mathrm{mg} / \mathrm{kg}$ per day. Steroid dosage was de creased and she was maintained on prednisolone $25 \mathrm{mg}$ on alternate days, azathioprine, and warfarin.

For the first 6 months after treatment renal function remained relatively stable, with a glomerular filtration rate of $18 \mathrm{ml} / \mathrm{min} / 1.73 \mathrm{~m}^{2}$. She continued to have proteinuria and mild hypertension which were controlled by prazosin and propranolol, and was able to return to school.

Six months after her initial recovery and despite $\alpha-G B M$ titre remaining undetectable, renal function began to decline. A repeat renal biopsy examination was performed in March 1980 to assess whether this deterioration was caused by continuing active disease, or by sclerosis of glomeruli damaged by the original insult. The biopsy specimen showed no evidence of active cellular proliferation, all glomeruli contained fibrous crescents, and there was extensive tubular atrophy and interstitial fibrosis. Immunofluorescence continued to show linear disposition of IgG. The appearances suggested an end stage crescentic nephritis with no evidence of active disease.

Despite the negative $\alpha$-GBM titres and the lack of histological evidence of active disease, 6 more plasmaphereses were performed. There was, however, no improvement in renal function which slowly declined, and continuous ambulatory peritoneal dialysis began 13 months after her original illness. 
Thus, despite her dramatic initial improvement after intensive treatment and the apparently inactive disease, permanent loss of renal function eventually occurred-presumably caused by sclerosis of glomeruli damaged by the original insult.

\section{Discussion}

These 3 children are among the first recorded cases of immunologically confirmed Goodpasture's syndrome in childhood, and the third child is also 1 of the few patients of any age with this disease to have recovered renal function once total anuria had occurred. Both these unusual events require explanation.

The lack of previous published reports of Goodpasture's syndrome in children may have been caused by failure of recognition rather than infrequent occurrence of the condition. Children thought to have post-streptococcal glomerulonephritis or crescentic nephritis of unknown aetiology may have had Goodpasture's syndrome and been misdiagnosed because of lack of awareness of its occurrence in childhood. Several reports of Goodpasture's syndrome in adults have commented on an apparent increase in incidence in recent years but this probably reflects increased awareness of the condition and facilities for more accurate diagnosis rather than a changing disease pattern. ${ }^{13}$ The same phenomenon may apply in children but this explanation does not adequately account for the lack of cases. Since immunofluorescence of renal biopsy specimens became routine in recent years and the linear deposition of immunoglobulin is unlikely to have been overlooked, alternative explanations should be considered.

Autoimmune disease is rare in childhood, and it is interesting that Anand et al. ${ }^{15}$ reported that the $\alpha$-GBM antigen that reacts with Goodpasture's sera is absent in young children. The children in cases 2 and 3 presented within 1 month of each other. In view of the known precipitation of Goodpasture's syndrome by hydrocarbons and viral infections an unidentified environmental agent may have been responsible. Other paediatric centres should be alert to the occurrences of the disease in childhood, as the disease pattern may be changing.

The low $C 3$ value in the child in case 2 is interesting as this is not usually a feature of Goodpasture's syndrome. The preceding upper respiratory infection, together with the initial low $\mathrm{C} 3$ value in this patient suggested a diagnosis of post-streptococcal glomerulonephritis. Streptococcal antigens cross react with GBM antigens, ${ }^{24}$ and intercurrent infections enhance any antibody mediated injury. ${ }^{25}$ A preceding streptococcal (or other) infection may therefore have been responsible for initiating or enhancing the $\alpha$-GBM mediated injury and bringing the case to light.

There are several possible reasons for the unusual recovery of renal function after 16 days anuria in the third patient. The kidney in childhood may have a greater potential for recovery than in adults but this explanation is inadequate as return of renal function was very closely associated with removal of $\alpha$-GBM antibody from the circulation. It is therefore likely that recovery was directly related to the effectiveness of treatment in removing he $\alpha$-GBM antibody.

The management of our third patient differed considerably from that of our first 2 , and of most reported cases in adults. Lack of awareness of the possibility of Goodpasture's syndrome in childhood resulted in both the first 2 patients undergoing a period of observation for presumed acute glomerulonephritis, and renal biopsy examination was only performed when continued deterioration of renal function occurred. The delay before referral, coupled with the period of observation and time spent awaiting the result of the renal biopsy examination resulted in a total delay of 2 months in the first patient and 2 weeks in the second, before treatment began. In addition to the delay before treatment lack of familiarity with the technical aspects of plasmapheresis in small ill children resulted in less effective treatment in these patients.

In contrast the diagnosis in the third patient was established and treatment with plasmapheresis, immunosuppression, and anticoagulation began within 24 hours of hospital armission. The availability of a rapid and sensitive :-GBM assay made quick diagnosis and treatment possible without first performing a renal biopsy examination. The speed with which measures to remove the $\alpha$-GBM began may have been a crucial factor in this patient's recovery.

The technical difficulties in performing plasmapheresis on small ill children had largely been overcome by the time our third patient was admitted. This enabled us to perform plasmapheresis rapidly and more intensively. The initial exchange of volumes equivalent on body weight basis to those used in most reported adult cases produced only a slow decrease in $\alpha$-GBM titre. Increasing the volume exchanged daily to 4 times the patient's total plasma volume resulted in a rapid drop of $\alpha$-GBM and a concurrent return of renal function. In addition to removing antibody, plasmapheresis may be beneficial in removing mediators of inflammation such as complement, fibrinogen, and kinins. The greater volumes exchanged would have produced more effective removal of these mediators 
and may also have played some role in the improved outcome.

If these explanations are correct and the recovery of renal function in the third child was due to more effective and rapid removal of the pathogenic antibody, a similar approach should be used in other patients. Although our patient eventually developed irreversible renal failure, her initial unexpected recovery suggests that earlier and more intensive treatment may produce complete recovery in other patients, particularly in those treated before extensive damage has occurred.

Because of the need to begin treatment quickly if permanent loss of renal function is to be averted, the diagnosis of Goodpasture's syndrome needs to be considered early in patients with nephritis with or without pulmonary symptoms. There are considerable logistic problems in measuring $\alpha$-GBM titres in all patients with glomerulonephritis but as the incidence of post-streptococcal nephritis has declined in most developed countries the rarer and more ominous forms of glomerulonephritis constitute a greater proportion of cases. Any child with an acute nephritic syndrome who has either severe renal dysfunction or who fails to follow the expected pathway to recovery should be referred urgently to a children's renal unit and be investigated immunologically and by renal biopsy examination as there are new methods of treatment that may avert permanent loss of renal function.

ML was supported by grants from the Kidney Research Aid Fund and the National Kidney Research Fund. SPAR was supported by the Kidney Research Aid Fund.

\section{References}

1 Stanton MC, Tange JD. Goodpasture's syndrome (pulmonary haemorrhage associated with glomerulonephritis). Austraiasian Annals of Medicine 1958;7:132-44.

2 Scheer RL, Grossman MA. Immune aspects of the g!omerulonephritis associated with pulmonary hemorrhage. Ann Intern Med 1964;60:1009-21.

3 Lerner RA, Glassock RJ, Dixon FJ. The role of antiglomerular basement membrane antibody in the pathogenesis of human glomerulonephritis. $J$ Exp Med 1967;126:989-1004.

4 Markowitz AS, Battifora HA, Schwartz F, Aseron C. Immunological aspects of Goodpasture's syndrome. Clin Exp Immunol 1968;3:585-91.

5 Wilson CB, Dixon FJ. Anti-glomerular basement membrane antibody induced glomerulonephritis. Kidney Int 1973;3:74-89.

6 Dunnhill MS. Goodpasture's syndrome. In: Dunnill MS, ed. Pathological basis of renal disease. London: WB Saunders, 1976:49-57.

7 Heptiristall RH. Lung hemorrhage and glomerulonephritis or Goodpasture's syndrome. In: Heptinstall RH, eds. Pathology of the kidney. 2nd ed. Boston: Little, Brown \& Co, 1974:580-99.

8 Koffler D, Sandson J, Carr R, Kunkel HG. Immunologic studies concerning the pulmonary lesions in Goodpasture's syndrome. Am J Pathol 1969;54:293-306.

9 Wilson CB, Dixon FJ. The renal response to immunologic injury. In: Brenner BM, Rector FC, eds. The kidney. Philadelphia: WB Saunders, $1981: 1237-350$.

10 Wilson CB, Marquardt H, Dixon FH. Radioimmunoassay (RIA) for circulating antiglomerular basement membrane (GBM antibodies) (Abstract). Kidney Int 1974;6:114A.

11 McPhaul JJ, Jr, Dixon FJ. The presence of anti-glomerular basement membrane antibodies in peripheral blood. $J$ Immunol 1969;103:1 168-75.

12 Sissons JGP, Evans DJ, Peters DK, et al. Glomerulonephritis associated with antibody to glomerular basement membrane. Br MedJ 1974;4:11-4.

13 Teague CA, Doak PB, Simpson IJ, Rainer SP, Herdson PB. Goodpasture's syndrome: an analysis of 29 cases. Kidney Int 1978;13:492-504.

14 Whitworth JA, Lawrence JR, Meadows R. Goodpasture's syndrome: a review of nine cases and an evaluation of therapy. Aust NZ J Med 1974;4:167-77.

15 Anand SK, Landing BH, Heuser ET, Olson DL, Grushkin CM, Lieberman E. Changes in glomerula basement membrane antigen(s) with age. $J$ Pediatr 1978; 92:952-3.

16 Siegler RL, Bond RE, Morris AH. Treatment of Goodpasture's syndrome with plasma exchange and immunosuppression. Clin Pediatr (Phila) 1980;19: 488-91.

17 Benoit FL, Rulon DB, Theil GB, Doolan PD, Watten RH. Goodpasture's syndrome, a clinicopathologic entity. $A m$ J Med 1964;37:424-44.

18 Beirne GJ. Goodpasture's syndrome and exposure to solvents. (Editorial). JAMA 1972;222:1555.

19 Rees AJ, Peters DK, Compston DAS, Batchelor JR. Strong association between HLA-DRW2 and antibodymediated Goodpasture's syndrome. Lancet 1978; : :966-8.

20 D'Apice AJF, Kincaid-Smith P, Becker GJ, Loughhead MG, Freeman JW, Sands JM. Goodpasture's syndrome in identical twins. Ann Intern Med 1978;88: 61-2.

21 Lockwood CM, Person TA, Rees AJ, Evans DJ, Peters DK, Wilson CB. Immunosuppression and plasmaexchange in the treatment of Goodpasture's syndrome. Lancet 1976; ; :711-5.

22 Rosenblatt SG, Knight W, Bannayan GA, Wilson CB, Stein JH. Treatment of Goodpasture's syndrome with plasmapheresis. Am J Med 1979;66:689-96.

23 Johnson JP, Whitman W, Briggs WA, Wilson CB. Plasmapheresis and immunosuppressive agents in antibasement membrane antibody-induced Goodpasture's syndrome. Am J Med 1978;64:54-9.

24 Markowitz AS, Lange CF, Jr. Streptococcal related glomerulonephritis. I. Isolation, immunochemistry and comparative chemistry of soluble fractions from type 12 nephritogenic streptococci and human glomeruli. $J$ Immunol 1964 ;92:565-75.

25 Rees AJ, Lockwood CM, Peters DK. Enhanced allergic tissue injury in Goodpasture's syndrome by intercurrent bacterial infection. Br Med J 1977;ii:723-26.

Correspondence to Dr Michael Levin, Renal Unit, Institute of Child Health, 30 Guildford Street, London WC1N 1EH.

Received 27 April 1983 